\title{
New Aspects of Diagnosis and Treatment of Sepsis
}

\section{Ostapiuk $L^{1,2 *}$}

${ }^{1}$ Department of Obstetrics and Gynaecology, Vinnytsia National Medical University of the Ministry of Health of Ukraine, Ukraine

${ }^{2}$ Lviv Regional Centre of Public Health, Lviv, Ukraine

*Corresponding Author: Ostapiuk Lesia, Vinnytsia National Medical University of the Ministry of Health of Ukraine, Lviv Regional Centre of Public Health, Lviv, Ukraine.

Since 1991, for the last 30 years, the world medical community has paid considerable attention to the problem of sepsis, one of the most serious diseases in the world with the high mortality rate. Significant work was done in order to understand the essence of this disease in order to develop effective methods of its treatment. The main attention was paid to the formulation of the definition of sepsis and its main diagnostic criteria. Three conciliatory world conferences (1991, 2001 and 2016) were dedicated to this problem. They offered important definitions and interesting views on the problem of sepsis. But, despite such big efforts of the world community, the problem of sepsis was not finally resolved. In this regard, in May 2017, the $70^{\text {th }}$ session of the World Health Assembly adopted a new resolution on sepsis on the basis of the report of the WHO Secretariat. According to it, the primary attention should be focused on improving early diagnosis, finding new markers and improving treatment tactics and monitoring of patients.

At the same time, since 2001, at the suggestion of Professor I. Herych (Lviv, Ukraine), a series of studies of blood serum (BS) of patients with purulent-inflammatory diseases and sepsis was started within the method of fluorescence spectroscopy (MFS). The main indicators evaluated were the fluorescence intensity $\left(\mathrm{I}_{F}\right)$ and the position of the fluorescence maximum $\left(\lambda_{\text {max }}\right)$. Very important results were obtained that helped to formulate the basic patterns of the behaviour of spectral-fluorescent characteristics of patients with sepsis. The behaviour of spectral-fluorescent characteristics in patients with sepsis during treatment was illustrated in the dynamics. For the in-depth understanding and explanation
Received: June 02, 2021

Published: June 15, 2021

(C) All rights are reserved by Ostapiuk L. of the patterns of the behaviour of spectral-fluorescent characteristics of BS, "in vitro disease models" were proposed, including the spectral-fluorescent model of sepsis in vitro. The dilution of BS with distilled water, sugar broth, $20 \%$ albumin solution, centrifuged and non-centrifuged bacterial culture (6-day culture of Staphylococcus aureus on sugar broth) were studied. This made it possible to simulate changes of the spectral-fluorescent characteristics of BS in various diseases and therapeutic measures. The fluorescence spectra (FS) of bacterial dilutions of BS make it possible to reproduce the proportions that are characteristic of FS of BS in patients with sepsis in vivo (when diluting BS by bacterial cultures, the fluorescence intensity $\left(\mathrm{I}_{F}\right)$ gradually decreases by more than $30 \%$ while increasing bacterial culture in solution. There is also the long-wave shift of the fluorescence bands $\left(\lambda_{\max }\right.$ ) of dilutions being higher than $10 \mathrm{~nm}$ ).

There were sufficient information about the pathogenetic factors of sepsis, but changes occurring at the molecular level are studied insufficiently. Their understanding is very important for understanding and developing the pathogenetic component of treating of purulent-inflammatory diseases and sepsis. Without the in-depth understanding of these aspects, the diagnosis and treatment of sepsis may notbeeffective enough. Especiallyimportant is the detoxification function of albumin, which is especially evident in patients with moderate endogenous intoxication. Due to the changes in the conformation of its molecules, albumin interacts with hydrophobic molecules of endotoxins and promotes their excretion from the body. In patients with purulent-inflammatory diseases and sepsis with increasing endogenous intoxication, most 
of the binding centers of albumin molecules are blocked by the products of bacterial metabolism. Although the total concentration of albumin in the blood of patients may be within normal limits, its actual "effective concentration" is much lower [1]. This leads to the violation of the basic functions of the body and requires timely implementation of effective treatment measures. In the case of the presence of low concentrations of toxins, albumin molecules are able to perform their detoxifying function. The pathogenetic concept of diagnostic and treatment model of purulent-inflammatory diseases and sepsis is proposed in this article in order to explain the changes that occur in the body in patients with sepsis. At the molecular level, it explains the changes that occur in this disease at the microscopic level. It is based on the fact that albumin molecules have the ability to complex. As a result, there are two types of albumin molecules in their blood: normal (concentration: $\mathrm{X}$ ) and blocked by toxins: pathological (concentration: $1-\mathrm{X}$ ). So, pathological albumin molecules lose the ability to perform their basic functions, namely transport and detoxification.

The new definition of sepsis is to define $X^{*}$, i.e. the maximal minimum value of the concentration of normal albumin molecules in the case of presence of a septic condition. If $\mathrm{X}$ is more than $\mathrm{X}^{*}$, this ensures the viability of the organism to some extent. In the case if $\mathrm{X}$ is less $\mathrm{X}^{*}$, exitus letalis develops.

These changes can be only registered with the help of MFS. After all, when conducting the biochemical blood test to determine the level of protein fractions, including albumin, we cannot separately record the presence of full-fledged or pathological albumin molecules. After all, a normal level of albumin in the blood is not the guarantee that all albumin molecules are normal, not blocked by toxins and can fully perform their basic functions.

Figure 1 and 2 illustrate spectral-fluorescent characteristics of patients with sepsis. The study of the FS of BS of the first patient (Figure 1) was conducted in the dynamics for more than 6 months until complete recovery of the patient. Curve 1 shows clearly the two-peak structure (in the region of $335 \mathrm{~nm}$ - barely noticeable "normal peak" and in the region of $380 \mathrm{~nm}$ - "septic peak"). The septic peak is associated with pathological albumin molecules and its magnitude is directly proportional to their number. Effective complex therapy led to the significant suppression of bacteremia and redistribution of the intensities of the above-mentioned fluorescent peaks. We were able to simulate the fluorescence curve of the BS of the mentioned patient on 02.01 (curve 1'). The right peak of this curve indicates a marked decrease in the concentration of pathological albumin molecules, and the left - an increase in the concentration of complete albumin in the patient's blood. Only a long process of treatment under the control of MFS led to the gradual suppression of bacteremia and recovery of the patient (Figure 1, curves 2-5). The nature of the spectral-fluorescent characteristics of this patient is consistent with the behaviour of the spectralfluorescent characteristics of the dilution of BS by bacterial culture in vitro [1]. When the patient's condition improves, the septic peak disappears, and the intensity of the normal peak increases and approaches the spectral-fluorescent characteristics of healthy individuals in the control group (donors).

Based on the pathogenetic concept of two varieties of albumin molecules in patients with sepsis, we proposed to use along with traditional treatment (surgical, etiotropic-antibiotic therapy, infusion therapy) as the pathogenetic treatment infusion of albumin solution in order to replenish the level of complete albumin in blood of patients with sepsis. This allows to support the vital functions of the patient's body. In case of the presence of the corresponding equipment, it is expedient to carry out the monitoring of the condition of patients by means of MFS. But even in the case of absence of appropriate equipment or the ability to conduct this study infusion of albumin solution will improve significantly the condition of patients, and ensure their recovery.

Figure 2 presents the results of the study of another person with sepsis. Due to the timely hospitalization and early surgical elimination of the source of infection, the progress of the septic process was much lower. Therefore, at the time of hospitalization there was only a significant decrease in the intensity of fluorescent spectra of BS, but the "septic" peak was absent, which was the good prognostic sign. The results of the study of FS of BS of this patient are in good agreement with the results of the in vitro studies [1].

Particular attention should be also paid to the urgency of the problem of sepsis for patients with diabetes. About $6 \%$ of albumin molecules in the BS of healthy donors are glycosylated. At the same time, 9 - 12\% of albumin molecules in patients with diabetes mellitus are glycosylated due to the presence of hyperglycemia. Therefore, patients with diabetes have the tendency to develop purulent-septic processes and their long-term course. It is logical that "sugar-laden albumin" is not able to bind completely and 


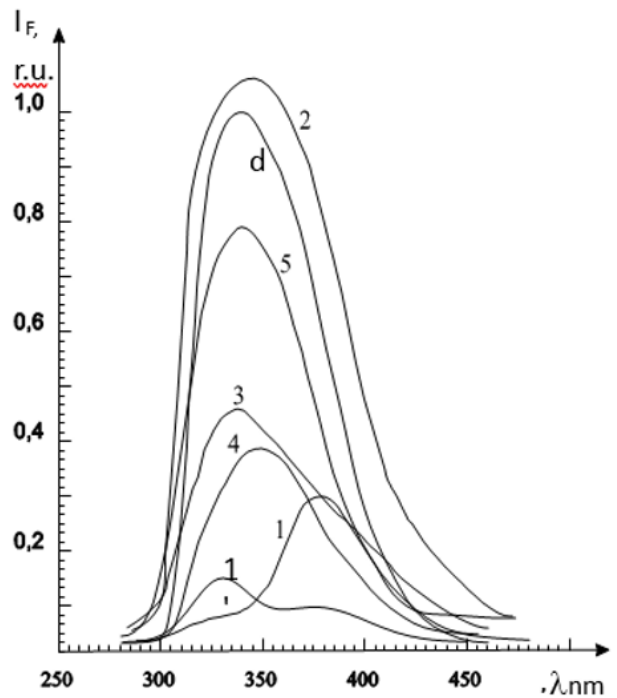

Figure 1: Fluorescence spectra of blood serum of patient 1 with sepsis: 1 - 28.12. ; 1'- 02.01. ; 2 - 04.01.; 3 - 12.02.; 4 - 19.03. 5 - 04.06. and donor of BS (335 nm - "normal peak", 380nm - "septic peak").

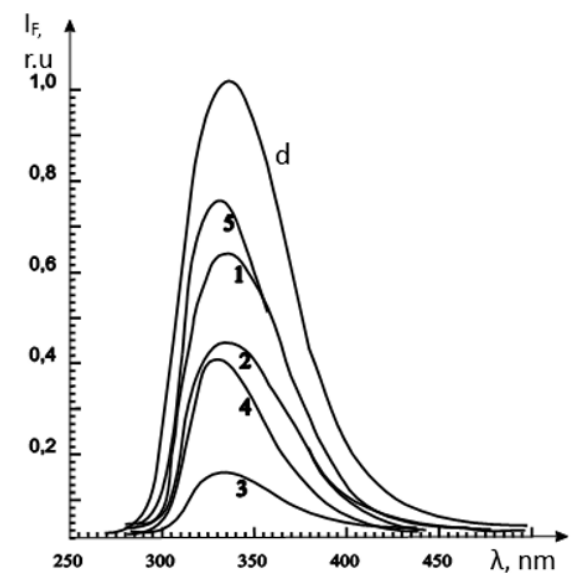

Figure 2: Fluorescence spectra of blood serum of patient 1 with sepsis: 1 - 3.06.; 2 - 05.06.; 3 - 06.06.; 4 - 07.06.; 5 - 10.06. and donor of BS. $\lambda \mathrm{ex}=280 \mathrm{~nm}$. eliminate toxic products from the body, which leads to a deepening of endogenous intoxication. FS of BS of the patient with sepsis and diabetes mellitus was presented in [1], This patient died due to the severe septic process.

The problem of sepsis is also relevant in obstetric practice. After all, postpartum purulent-inflammatory diseases are the significant cause of maternal mortality, especially in low-income countries. It is important to monitor the condition of women in the postpartum period, especially with the use of MFS [2], and the timeliness of medical care in the development of postpartum complications. Let us consider this on the example of postpartum endometritis. The postpartum endometritis was diagnosed during MFS monitoring (I ${ }_{F} \leq 0,35$ r.u.). After stabilization of the patient's condition manual vacuum aspiration of the uterine cavity walls, antibiotic therapy, uterotonic therapy, were performed. After applying this treatment, the patient's condition improves, and she recovered.In the case of absence of timely monitoring within the MFS or in the case of its underestimation without MFS, this can lead to the significant deterioration of the patient's condition. The timely correction of the treatment process (manual vacuum aspiration of the walls of the uterine cavity, antibiotic therapy, anti-inflammatory and infusion therapy), including infusion of albumin solution (repeatedly), still gives the chance for their full recovery. At the subsequent postponement of operative treatment development of obstetric sepsis with the unfavorable forecast is expected.

Obtaining the information about the mechanisms of origin and evolution of purulent-inflammatory diseases and sepsis is extremely important for the rapid search for the effective ways to treat them. This is why we have proposed the pathogenetic concept of diagnostic and therapeutic approach for purulent-inflammatory diseases and sepsis. Within the MFS, thorough studies of the spectral-fluorescent characteristics of BS of patients with postpartum purulent-inflammatory diseases, patients with surgical profile and burn injury as well as sepsis were conducted. It is established that the studied characteristics were universal markers of the severity of the patients' condition. At the same time their changes were registered for 24 - 48 hours before emergence of obvious clinical and laboratory signs of the general somatic condition of patients $[1,2]$.

\section{Bibliography}

1. Ostapiuk L. "Diagnostic and Therapeutic Model of Sepsis and Purulent-Inflammatory Diseases". International Journal of Clinical Medicine 10 (2019): 577-595. 
New Aspects of Diagnosis and Treatment of Sepsis

2. Bulavenko O., et al. "Problems and Challenges to Women's Reproductive Health in the 21th Century". Acta Scientific Women's Health 3 (2021): 70-87.

Volume 3 Issue 7 July 2021

(c) All rights are reserved by Ostapiuk $L$. 